\title{
Immunotherapy for metastatic urothelial carcinoma: putting the brakes on releasing the brake
}

\author{
Matthew Zibelman ${ }^{1}$ \& Elizabeth R. Plimack*,1 \\ ${ }^{1}$ Hematology/Oncology, Fox Chase Cancer Center, Temple Health, 333 Cottman Ave, Philadelphia, PA 19111, USA \\ * Author for correspondence: elizabeth.plimack@fccc.edu
}

"The excitement of ICB must be tempered by its limitations. Progress remains on the horizon, as biomarkers and combinations provide hope that clinicians will be better able to select therapies that will optimize efficacy and minimize toxicity at the individual patient level."

First draft submitted: 15 January 2018; Accepted for publication: 16 March 2018; Published online: 22 March 2018

Systemic treatment for metastatic urothelial carcinoma (mUC) has encountered a therapeutic renaissance with the approval of five new agents by the US FDA since 2016. Prior to that, no drug or combination had established a definitive therapeutic foothold since the investigation of taxanes and cisplatin-based regimens (gemcitabine/cisplatin [GC] or accelerated methotrexate/vinblastine/adriamycin/cisplatin [aMVAC]) in the early 2000s. For first-line therapy, cisplatin-eligible patients can achieve some of the highest response rates in oncology - 72\% with aMVAC - but with clinically significant toxicity and few clear options in the second-line and beyond for relapsing patients. Patients unable to safely be treated with cisplatin encountered a therapeutic nihilism rooted in the reality that the few available therapies yielded minimal efficacy and limited durable benefit. The emergence of immune checkpoint inhibitors targeting the PD-1 has dramatically altered the therapeutic landscape for these patients, offering the promise of a durable response in a subset of patients in both cisplatin-eligible and ineligible populations, and with an overall more favorable toxicity profile. Yet, immunotherapy for bladder cancer and other UCs of the genitourinary tract is not a panacea, as it remains limited by a lack of clinically validated predictive biomarkers, capable of resulting in severe and persistent immune-related adverse events (irAEs) and is unlikely to completely replace the formidable presence of cisplatin-based chemotherapy.

Immune checkpoint blockade (ICB) targeting the PD-1 pathway (either the receptor found predominantly on immune cells or the cognate ligand primarily on tumor cells) results in a non-specific immune modulation that re-energizes exhausted effector T-cells, previously unable to mount an anti-tumor response due to tumor-directed defense mechanisms, subsequently rendered immune-competent [1]. The lay refrain becoming increasingly familiar to most medical oncologists is that these drugs 'release the brake' on the immune system, letting the patient's own immune mechanisms ostensibly perform the job they were designed for, but which had been hijacked by signals stemming from the tumor's own struggle for survival. It's an elegant mechanism that can work brilliantly for some patients, is tailor-made for direct to consumer advertising, and speaks to a populace clamoring for less toxic therapies and ways to enhance our own innate systems. However, it is incumbent upon medical oncologists to also understand the limitations and risks associated with these therapies and advise patients honestly regarding available data in order to set realistic expectations.

Five anti-PD1 pathway inhibitors (atezolizumab, nivolumab, durvalumab, avelumab and pembrolizumab) have been approved by the US FDA for the treatment of patients with platinum-refractory mUC, which includes patients who develop recurrent metastatic disease within 12 months of receiving neoadjuvant or adjuvant cisplatinbased chemotherapy. At this time, only pembrolizumab is supported by level one evidence (KEYNOTE-045 trial, NCT02256436) demonstrating an overall survival benefit compared to physicians choice chemotherapy (either paclitaxel, docetaxel, or vinflunine outside of the USA) in a randomized Phase III trial [2]. Thus, until such time that more data becomes available, or trials exist comparing efficacy or toxicity between these agents, pembrolizumab should be considered the standard-of-care for this indication. In KEYNOTE-045, pembrolizumab yielded a median 
overall survival (mOS) of 10.3 months (versus 7.4 months with chemotherapy; $\mathrm{p}<0.01$ ) and an overall response rate (ORR) of $21 \%$ in all patients. Despite a somewhat modest ORR, the true benefit of ICB is the durability in responders, the so-called 'tail of the (Kaplan-Meier) curve', and accordingly at a median follow-up of 14.1 months, the 12 month landmark OS was $43.9 \%$, compared to only $30.7 \%$ in patients receiving chemotherapy, with $72 \%$ of pembrolizumab responders maintaining at least a PR at data cutoff. Thus, the majority of patients do not benefit, however for those that do, prolonged response and survival is possible. Continued long-term follow-up of this study will be important in order to further understand the clinical destiny of these patients.

Since only a minority of patients who receive ICB benefit, biomarkers that can predict response, or lack thereof, are in demand. PD-L1 expression remains elusive as a standalone marker, limited by variability in assays used, testing cut-offs, cell types assessed (tumor cells vs immune cell vs both) and likely by the pure fact that the mechanisms underlying response to ICB are complicated and a single marker is unlikely to yield a negative predictive value robust enough to deny treatment. Next-generation biomarkers are promising and a source of intense study, but not yet validated to inform clinical decision making. Effective immunotherapy requires at a minimum a tumor microenvironment (TME) penetrable by effector T-cells, an immunotolerant metabolic milieu, and available tumor antigens recognizable to effector T-cells. Thus, strategies to assess for, or serve as surrogates for, these conditions hold promise as more relevant biomarkers. In the Phase II IMvigor 210 study of atezolizumab in platinum-refractory metastatic UC, response was associated with higher expression of IFN $\gamma$-inducible cytokines CXCL9 and CXCL10, consistent with a more immunologically active TME, and also with greater CD8+ T-cell infiltration [3]. Tumor mutational load (TML) has also been investigated as a potential immunotherapy biomarker in $\mathrm{mUC}$, as well as in other tumor types [3-5]. Further study will help delineate whether a high TML itself characterizes a more genetically altered and immunogenic phenotype, or whether it is merely a surrogate marker for tumors that have more chances to have 'the right' mutations that lead to relevant tumor antigens. An IFN $\gamma$ signature has also been explored in $\mathrm{mUC}$ to predict response, utilizing the knowledge that IFN $\gamma$ is a driver of PD-L1 expression, and that its presence is essential for response to ICB [6]. Several studies of PD-1 blockade in mUC have demonstrated an association with response and an enhanced IFNg signature, but standardization of the variables and platform used will be important in future validation of this marker in pursuit of clinical relevance $[7,8]$.

Compared to cytotoxic chemotherapy, on the whole ICB targeting the PD-1 pathway has a better toxicity profile and leads to an improved quality of life for patients [9]. However, ICB can lead to serious and even fatal irAEs and treating clinicians must remain vigilant when assessing for toxicity, as serious irAEs may present in subtle and variable patterns. Most chemotherapy-associated toxicity is transient and resolves once the drugs are discontinued, but irAEs to ICB can be long-lasting, may require long steroid tapers or life-long replacement supplementation (e.g., hydrocortisone for adrenal insufficiency) and can occur even after stopping treatment. Pneumonitis, myocarditis, endocrinopathies, Stevens-Johnson syndrome, and various neurologic sequelae have been reported and can be fatal or lead to lifelong debilitating morbidity [10-13]. Additionally, non-oncologists may not be familiar with these drugs and their unique toxicity profiles, thus patients presenting to primary care providers with new symptoms may not always get consideration for steroid-based immunosuppressive therapy if needed. Thus, appropriate informed consent, as well as patient and staff education are integral to ensure potential irAEs are recognized and managed expeditiously.

Undoubtedly, ICB is now an important part of the systemic therapy landscape for mUC, but how it will ultimately integrate itself into the treatment paradigm remains uncertain. In its current FDA-approved settings, for cisplatin-refractory or ineligible patients, ICB is the clear choice for most patients, but these settings had no clear option previously, as various single agent chemotherapy drugs have led to modest, often short-lived benefit [14]. ICB is now being studied in the first-line cisplatin-eligible setting, both with and without chemotherapy, but this may prove to be a much harder hurdle to clear. In the follow-up to the landmark Phase III trial comparing aMVAC to classic MVAC, the five-year survival rate in the aMVAC arm was $21.8 \%$, demonstrating that a subset of patients do derive long-term benefit from cisplatin-based chemotherapy and pins a tail on the OS curve that may be similar to that seen with ICB [15]. Furthermore, emerging data reveal that tumors with defects in certain DNA repair genes may predict response to cisplatin-based chemotherapy in the neoadjuvant setting, and this may hold true for metastatic patients as well $[16,17]$. Combined ICB (adding a CTLA- 4 inhibitor to a PD-1 inhibitor) has demonstrated improved efficacy over either drug alone in other malignancies (melanoma, non-small cell lung cancer, renal cell carcinoma albeit with greater toxicity, but whether this will prove superior to effective cisplatinbased chemotherapy for mUC remains to be seen [18-20]. Other combinatorial strategies, including adding vaccines, 
oncolytic viruses, or indoleamine-2,3-dioxygenase inhibitors to anti-PD-1 drugs, may also be viable, and potentially with less toxicity than with combined ICB.

In the world of mUC, the past 2 years have been a whirlwind of drug approvals and promise for better patient outcomes. However, work remains to be done, as too few patients respond, and we cannot yet accurately predict who is most likely to benefit. The excitement of ICB must be tempered by its limitations. Progress remains on the horizon, as biomarkers and combinations provide hope that clinicians will be better able to select therapies that will optimize efficacy and minimize toxicity at the individual patient level. Until then, when talking to patients about 'releasing the brakes' on the immune system, we should be sure to tap the brake ourselves.

\section{Financial \& competing interests disclosure}

Matt Zibelman has Institutional research Support: BMS, Horizon Pharma, Grants: Pfizer, Consulting: EMD Serono, Horizon Pharma. Elizabeth Plimack has Consulting/Scientific Advisory: AstraZeneca, BMS, Genentech, Novartis, Roche, Horizon Pharma, Inovio, Merck, Clovis. has Research Study Grants from AstraZeneca, Bristol-Myers Squibb, Dendreon, Eli Lilly Inc., Merck, Pfizer. The authors have no other relevant affiliations or financial involvement with any organization or entity with a financial interest in or financial conflict with the subject matter or materials discussed in the manuscript apart from those disclosed.

No writing assistance was utilized in the production of this manuscript.

\section{References}

1 Pardoll DM. The blockade of immune checkpoints in cancer immunotherapy. Nat. Rev. Cancer. 12(4), 252-264 (2012).

2 Bellmunt J et al. Pembrolizumab as second-line therapy for advanced urothelial carcinoma. N. Engl. J. Med. 376(11), 1015-1026 (2017).

3 Rosenberg JE et al. Atezolizumab in patients with locally advanced and metastatic urothelial carcinoma who have progressed following treatment with platinum-based chemotherapy: a single-arm, multicentre, Phase 2 trial. The Lancet (2016).

4 Snyder A et al. Genetic Basis for Clinical Response to CTLA-4 Blockade in Melanoma. N. Engl. J. Med. 371(23), 2189-2199 (2014).

5 Goodman AM et al. Tumor mutational burden as an independent predictor of response to immunotherapy in diverse cancers. Mol. Cancer Ther. (2017).

6 Taube JM et al. Colocalization of inflammatory response with B7-h1 expression in human melanocytic lesions supports an adaptive resistance mechanism of immune escape. Sci. Trans. Med. 4(127), 127ra37-127ra37 (2012).

7 Bais C et al. Biologic and clinical relevance of an IFNG mRNA signature (IFNGS) and PD-L1 protein expression in tumor and immune cells in urothelial cancer (UC) patients (pts) treated with durvalumab (D). J. Clin. Oncol. 35(15_suppl), 3037-3037 (2017).

8 O'Donnell PH et al. Biomarker findings and mature clinical results from KEYNOTE-052: First-line pembrolizumab (pembro) in cisplatin-ineligible advanced urothelial cancer (UC). J. Clin. Oncol. 35(15_suppl.), 4502-4502 (2017).

9 Vaughn DJ et al. Health-related quality of life (HRQoL) in the KEYNOTE-045 study of pembrolizumab versus investigator-choice chemotherapy for previously treated advanced urothelial cancer. J. Clin. Oncol. 35(6_suppl), 282-282 (2017).

10 Johnson DB et al. Fulminant myocarditis with combination immune checkpoint blockade. N. Engl. J. Med. 375(18), 1749-1755 (2016).

11 Michot J et al. Immune-related adverse events with immune checkpoint blockade: a comprehensive review. Eur. J. Cancer. 54, 139-148 (2016).

12 Plimack ER et al. Endocrine-Related Adverse Events Associated With Immune Checkpoint Blockade and Expert Insights on Their Management. (2017).

13 Zimmer L et al. Neurological, respiratory, musculoskeletal, cardiac and ocular side-effects of anti-PD-1 therapy. Eur. J. Cancer 2016(60), 210-225 (2016).

14 Zibelman M, Plimack ER. Systemic therapy for bladder cancer finally comes into a new age. Future Oncol. 12(19), 2227-2242 (2016).

15 Sternberg CN et al. Seven year update of an EORTC Phase III trial of high-dose intensity M-VAC chemotherapy and G-CSF versus classic M-VAC in advanced urothelial tract tumours. Eur. J. Cancer. 42(1), 50-54 (2006).

16 Plimack ER et al. Defects in DNA repair genes predict response to neoadjuvant cisplatin-based chemotherapy in muscle-invasive bladder cancer. Eur. Urol. 68(6), 959-967 (2015).

17 Van Allen EM et al. Somatic ERCC2 mutations correlate with cisplatin sensitivity in muscle-invasive urothelial carcinoma. Cancer Discov. 4(10), 1140-1153 (2014).

18 Postow MA et al. Nivolumab and ipilimumab versus ipilimumab in untreated melanoma. N. Engl. J. Med. 372 (20175).

19 Hellmann MD et al. Nivolumab plus ipilimumab as first-line treatment for advanced non-small-cell lung cancer (CheckMate 012): results of an open-label, Phase 1, multicohort study. Lancet Oncol. 18(1), 31-41 (2017).

20 Escudier B et al. LBA5CheckMate 214: Efficacy and safety of nivolumab+ ipilimumab (N+ I) v sunitinib (S) for treatment-naïve advanced or metastatic renal cell carcinoma (mRCC including IMDC risk and PD-L1 expression subgroups. Ann. Oncol. 28(suppl_5) (2017). 
